\title{
The Radius of Curvature of the Parabola in Two Diminutions
}

\author{
Abdel Radi Abdel Rahman Abdel Gadir Abdel Rahman', Eman Farah Altayb Alsamani², \\ Neama Yahia Mohammed ${ }^{3}$ \\ ${ }^{1,2}$ Department of Mathematics, Faculty of Education, Omdurman Islamic University, Omdurman, Sudan \\ ${ }^{3}$ Department of Mathematics, College of Science, Tabuk University, Saudi Arabia
}

\begin{tabular}{ll}
\hline ARTICLE INFO & ABSTRACT \\
\hline Published online & $\begin{array}{l}\text { In the present paper we dealt with some aspects of Curves, curvature and Radius of curvature. The } \\
\text { aim of this study lies in the Radius of Curvature of the Parabola in 2-dimenssions numerically and }\end{array}$ \\
$\begin{array}{ll}\text { Corresponding author: } \\
\text { using Matlab. Matlab is an interactive working environment in which user can carry out quite complex } \\
\text { Abdel Radi Abdel }\end{array}$ & $\begin{array}{l}\text { computational tasks with few commands. We followed the applied mathematical method using a new } \\
\text { mathematical technique (Matlab) and we found that the Radius of curvature of the Parabola in tow } \\
\text { Rahman Abdel Gadir } \\
\text { diminutionus using a new mathematical technique is more accurate and speed than the numerical } \\
\text { calculating. }\end{array}$ \\
\hline KEYWORDS: Curve, Curvature, Radius of Curvature, Parabola, Two Diminutions, Matlab.
\end{tabular}

\section{INTRODUCTION}

Radius of curvature $\mathrm{R}$ is the reciprocal of the curvature in differential geometry. It is the radius of the circular arc that best approximates the curve at the point for a curve. The radius of curvature of a surface is the radius of a circle that best matches a normal section or combinations of normal sections. Curvature is a numerical measure of bending of the curve. At a particular point on the curve a tangent can be drawn. Let this line makes an angle $\Psi$ with positive $\mathrm{x}$ - axis. Then curvature is defined as the magnitude of rate of change of $\Psi$ with respect to the arc length s.Curvature at

$P=\left|\frac{d \Psi}{d s}\right|$. It is obvious that smaller circle bends more sharply than larger circle and thus smaller circle has a larger curvature.

Radius of curvature is the reciprocal of curvature and it is denoted by $\rho$.

Matlab is a powerful computing system for handling the calculations involved in scientific and engineering problems. The name Matlab stands for Matrix Laboratory because the system was designed to make matrix computations particularly easy.

One of the many things you will like about Matlab (and which distinguishes it from many other computer programming systems such as $\mathbf{C}^{++}$and Java) is that you can use it interactively. This means you type some commands at the special Matlab prompt and get the answers immediately. The problems solved in this way can be very simple like finding a square root or they can be much more complicated like finding the solution to a system of differential equations.[3,pp3]

Matlab is a computer programming language used in engineering, science and applied mathematics. It comes with a robust programming language, stunning graphics, and a wealth of expert knowledge. A trademark of the math works publishes Matlab. Matlab focuses on computing rather than mathematics: Symbolic operations and expressions are not possible (except through the optional symbolic tool box, a clever interface to Maple). All of the results are not only numerical, but also approximate. The limitation of numerical computing can be viewed as a disadvantage, but it can also be viewed as a strength: When it comes to numeric Matlab is far superior to Maple, Mathematic, and other similar programs. Matlab on the other hand, is considerably easier to use than other numerically oriented languages such as $\mathrm{C}^{++}$and Fortran.

\section{CURVES}

In simple terms a space curve is a set of connected points in the space such that any totally-connected subset of it can be twisted into a straight line segment without affecting the neighborhood of any point. More technically, a curve is defined as a differentiable parameterized mapping between an interval of the real line and a connected subset of the space that is $C(t): I \rightarrow R^{3}$ where $C$ is a space curve defined on the interval $I \subseteq R$ and parameterized by the variable $t \in I$. Hence different parameterizations of the same 
"geometric curve" will lead to different "mapping curves". The image of the mapping in $R^{3}$ is known as the trace of the curve; hence different mapping curves can have identicaltraces. The curve may also be defined as a topological image of a real interval and maybe linked to the concept of Jordan arc.[14,pp25]

Definition (2.1): Given $k \in N \cup\{\infty\}$ and $n \geq 2$ a parameterized curve of class $C^{k}$ in $R^{n}$ is a map $\sigma: \mathrm{I} \rightarrow \mathrm{R}^{\mathrm{n}}$ of class $C^{\mathrm{k}}$ where $I \subseteq R$ is an interval.

The image $\sigma(I)$ is often called support (or trace) of the curve; the variable $t \in I$ is the parameter of the curve. If $I=[a, b]$ and $\sigma(a)$ $=\sigma(b)$ we shall say that the curve is closed. [12,pp4]

Definition (2.2): A simple closed plane curve $\gamma:[a, b] \rightarrow R^{2}$ is called positively oriented if it satisfies the following equivalent conditions:

i. The rotation index of $\gamma$ equals 1 .

ii. The interior is on one's left as one traverses $\gamma$ more precisely for each $\mathrm{t} \in[\mathrm{a}, \mathrm{b}], \mathrm{s} R_{90}\left(\gamma^{\prime}(\mathrm{t})\right)$ points toward the interior in the sense that there exists $\delta>0$ such that

$\gamma(\mathrm{t})+\mathrm{s} R_{90}\left(\gamma^{\prime}(\mathrm{t})\right)$ lies in the interior for all $\mathrm{s} \in(0, \delta)$.

Otherwise $\gamma$ is negatively oriented in which case its rotation index equals -1 and $R_{90}\left(\gamma^{\prime}(\mathrm{t})\right)$ points toward the exterior for all $\mathrm{t} \in[\mathrm{a}$, b].[10,pp62]

\section{Definition (2.3):}

A parameterized curve $\tilde{\gamma}: J \rightarrow R^{n}$ is a reparameterization

of $\gamma: I \rightarrow R^{n}$ if there is a diffeomorphism $\emptyset: \mathrm{J} \rightarrow \mathrm{I}$ such that $\tilde{\gamma}(\tilde{t})=\gamma_{\circ} \emptyset(\tilde{t})$ for all

$\tilde{t} \in \mathrm{J}$.

Definition (2.4):

A point $\gamma(t)$ is called a regular point if $\gamma^{\prime}(t) \neq 0$; otherwise

$\gamma(t)$ is a singular point of $\gamma$. A curve is regular if all points are regular.[17,pp10]

\section{Definition (2.5):}

A differentiable curve is a $C^{\infty}$ map: $\mathrm{I} \rightarrow R^{3}$ defined on an open possibly unbounded interval $I$ of $R$. Referring to a vector-valued function $\alpha$ the word differentiable means that if :

$\alpha(t)=(x(t), y(t), z(t))$

the three coordinate functions $x, y, z$ are differentiable. The vector

$a^{\prime}(t)=\left(x^{\prime}(t), y^{\prime}(t), z^{\prime}(t)\right)$

whose components are the derivatives of the components of $\alpha$ is called thetangent vector or velocity vector of the curve $\alpha$ at $t \in I$ or at the point $\alpha(\mathrm{t})$ even though this last expression is ambiguous. We say that the curve $\alpha: I \rightarrow R^{3}$ is a plane curve if there exists a plane $P \subset R^{3}$ such that $\alpha(I) \subset P$. Since we are considering only those properties of curves that are invariant under rigid motions and we can always find a rigid motion of $R^{3}$ taking the plane $\mathrm{P}$ to be the plane $z=0$, we may restrict our selves to the case

$\alpha: I \rightarrow R^{3}$ where:

$\alpha(t)=(x(t), y(t), 0)$

that is to the case of differentiable maps $\alpha: I \rightarrow R^{2}$.[13,pp3]

Theorem (2.6):

If a is a regular curve in $\mathbf{R}^{3}$ then there exists a reparameterization $\beta$ of $\alpha$ such that $\beta$ has unit speed.

Proof. Fix a number $a$ in the domain $I$ of $\alpha: I \rightarrow \boldsymbol{R}^{3}$ and consider the arc length function:

$s(t)=\int_{a}^{b}\left\|\alpha^{\prime}(u)\right\| d u$

(The resulting reparameterization is said to be based at $t=a$.) Thus the derivative $d s / d t$ of the function $s=s(t)$ is the speed function $v=\left\|\alpha^{\prime}\right\|$ of $\alpha$. Since $\alpha$ is regular by definition $\alpha^{\prime}$ is never zero; hence $d s / d t>0$. By a standard theorem of calculus the function $s$ has an inverse function

$t=t(s)$ whose derivative $d t / d s$ at $s=s(t)$ is the reciprocal of at

$t=t(s)$. In particular $d t / d s>0$.

Now let $\beta$ be the reparametrization $\beta(s)=\alpha(t(s))$ of $\alpha$. We assert that $\mathrm{b}$ has unit speed. In fact :

The speed of $\beta$ is :

$$
\beta^{\prime}(s) \frac{d t}{d s}(s) \alpha^{\prime}(t(s))
$$

Definition (2.7):

$$
\left\|\beta^{\prime}(s)\right\|=\frac{d t}{d s}(s)\left\|\alpha^{\prime}(t(s))\right\|=\frac{d t}{d s}(s) \frac{d s}{d t}(t(s))=1 .
$$

A vector field $Y$ on curve $\alpha: I \rightarrow \boldsymbol{R}^{3}$ is a function that assigns to each number $t$ in I a tangent vector $Y(t)$ to $\boldsymbol{R}^{3}$ at the point $\alpha(t) \cdot[2, \mathrm{pp} 53]$

Theorem (2.8):

(The Frenet Formulas for Non-Unit Speed Curves). Fora regular curve $\alpha(\mathrm{t})$ with speed $v=d s / d t$ and curvature $k>0$ 
$T^{\prime}(t)=k v N$

$$
N^{\prime}(t)=-k v T+\tau v B
$$

$B^{\prime}(t)=-\tau v N$

Proof: The unit tangent $T(t)$ is $\bar{T}(s)$ by definition. Now $T^{\prime}(t)$ denotes differentiation with respect to t so we must use the chain rule on the right-hand side to determine $k$ and $\tau$.

$T^{\prime}(t)=\frac{d \bar{T}(s)}{d s} \frac{d s}{d t}$

$$
\begin{aligned}
& =k^{\prime}(s) N(s) v \\
& =k(t) N(t) v(t) \quad \text { by definition } \\
T^{\prime} & =k v N,
\end{aligned}
$$

so the first formula is proved. For the second and third

$$
\begin{aligned}
N^{\prime}(t) & =\frac{d \bar{N}(s)}{d s} \frac{d s}{d t} \\
& =(-\bar{k} \bar{T}+\bar{\tau} \bar{B}) \mathrm{V}
\end{aligned}
$$

by the unit speed Frenet formulas:

$$
=-k v T+\tau v B \text {. }
$$

and

$$
\begin{aligned}
B^{\prime}(t) & =\frac{d \bar{B}(s)}{d s} \frac{d s}{d t} \\
& =-\bar{\tau} \bar{N} v \\
& =-\tau v N . \quad[9, \mathrm{pp} 28])
\end{aligned}
$$

\section{Theorem(2.9):}

(Plane curves with constant curvature)

A regular curve in $R^{2}$ has constant curvature $k$ if and only if it is part of a circle of radius $\frac{1}{|k|}$ (if $k \neq 0$ ) or a line segment (if $k=0$ ).

Proof: The proof follows directly from the Frenet equations. Assume first that

$k\left(s_{0}\right)=0$ for a fixed $s_{0}$. Obviously the expression $c(s)+\frac{1}{k\left(s_{0}\right)} e_{2}(s)$ is constant if and only if $c(s)$ is part of a circle of radius $\left|\frac{1}{k\left(s_{0}\right)}\right|$ since the difference vector has constant length $\left|\frac{1}{k\left(s_{0}\right)}\right|$.

This is equivalent to the constancy $k=k\left(s_{0}\right)$ everywhere because :

$\mathrm{c}^{\prime}+\frac{1}{k\left(s_{0}\right)} e_{2}^{\prime}=1-\frac{1}{k\left(s_{0}\right)} k e_{1}$.

The fact that $k \equiv 0$ only holds for line segments follows from $e_{2}^{\prime}=-k e_{1}$ : The condition $0=k e_{2}(s)=e_{1}^{\prime}(s)=c^{\prime \prime}(s)$ directly implies $c^{\prime}(s)=\mathrm{a}$ and $c(s)=s \mathrm{a}+\mathrm{b}$ with constant $\mathrm{a}, \mathrm{b} \in R^{2}$. [18,pp15]

\section{Proposition (2.10):}

A space curve is planar if and only if its torsion is everywhere 0 . The only planar curves with nonzero constant curvature are (portions of) circles.

Proof: If a curve lies in a plane $P$, then $T(s)$ and $N(s)$ span the plane $\mathrm{P}_{0}$ parallel to $P$ and passing through the origin. Therefore $B=$ $T \times N$ is a constant vector (the normal to $P_{0}$ ) and so $B^{\prime}=-\tau N=0$ from which we conclude that $\tau=0$. Conversely if $\tau=0$ the bi normal vector $B$ is a constant vector $B_{0}$. Now consider the function

$\mathrm{f}(\mathrm{s})=(s) . B_{0}$ we have $f^{\prime}(s)=\alpha^{\prime}(s) \cdot B_{0}=T(s) \cdot B(s)=0$ and so $f(s)=c$ for some constant $c$. This means that $\alpha$ lies in the plane $x s . B_{0}=c$.

That a circle of radius $a$ has constant curvature 1/a . Now suppose a planar curve $\alpha$ has constant curvature $k_{0}$. Consider the auxiliary function $\beta(s)=\alpha(s)+\frac{1}{k_{0}} N(s)$. Then we have :

$$
\beta^{\prime}(s)=\alpha^{\prime}(s)+\left(-k_{0}(s) T(s)\right)=T(s)-T(s)=0 .
$$

Therefore $\beta$ is a constant function say $\beta(s)=p$ for all $s$. Now we claim that $\alpha$ is a (subset of a) circle centered at $P$ for :

$$
\left.\|\alpha(s)-p\|=\|\alpha(s)-\beta(s)\|=1 / k_{0} .[15, \mathrm{pp} 15]\right)
$$

\section{CURVATURE}

The curvature of a curve is a useful entity, so it deserves to be rigorously defined.

Intuitively the curvature should be a number that measures how much the curve deviates from a straight line at any point. It should be large in areas where the curve wiggles oscillates or makes a sudden direction change; it should be small in areas where the curve is close to a straight line. It is also useful to associate a direction with the curvature i.e. to make it a vector. Given a parametric curve $P(t)$ and a point $P(i)$ on it, we calculate the first two derivatives $P^{t}(i)$ and $P^{t t}(i)$ of the curve at the point. We then construct a circle that has these same first and second derivatives and move it so it grazes the point. This is called the osculating circle of the curve at 
the point. The curvature is now defined as the vector $k(i)$ whose direction is from point $P(i)$ to the center of this circle and whose magnitude is the reciprocal of the radius of the circle. Using differential geometry it can be shown that the vector: $\frac{p^{t}(t) \times p^{t t}(t)}{\left|p^{t}(t)\right|^{3}}$ has the right magnitude. However, this vector is perpendicular to both $P^{t}(t)$ and $P^{t t}(t)$, so it is perpendicular to the osculating plane.[5,pp30]

\section{Definition (3.1):}

Let $C$ be a regular curve parametrized by $\vec{x}: I \rightarrow \mathrm{R}^{2}$

with curvature function $k_{g}(t)$. A vertex of the curve $C$ is a point

$P=\vec{x}\left(t_{0}\right)$ where $k_{g}\left(t_{0}\right)=0 .[16, \mathrm{pp} 25]$

\section{Definition (3.2):}

Let $f(s)$ be a normal representation of class $C^{2}$ of a plane curve; write $k(s)$ for its curvature at the point with parameter $s$. The relative curvature with respect to this normal representation $f$ is the quantity :

i. 0 if $f^{\prime \prime}(s)=0$;

ii. $+k(s)$ if $\left(f^{\prime}(s), f^{\prime \prime}(s)\right)$ is a basis having the same orientation as the canonical

basis of $\mathrm{R}^{2}$;

iii. $-k(s)$ if $\left(f^{\prime}(s), f^{\prime \prime}(s)\right)$ is a basis having the orientation opposite to that of the canonical basis of $\mathrm{R}^{2}$.[6,pp92]

Definition (3.3):

Let $\mathrm{M} \subseteq \mathbf{R}^{3} n$ be anon degenerate regular surface. If its mean

Curvature vanishes, we say that the surface is critical. In particular $M$ is minimal if

$\mathbf{M} \subseteq \mathbf{R}^{3}$ or if it is time like and maximal if $M \subseteq \mathbf{L}^{3}$ is space like.[7,pp201]

\section{Definition (3.4):}

Let $\sigma: I \rightarrow R^{n}$ be a regular curve of class $\mathrm{C}^{\mathrm{k}}$ (with $\mathrm{k} \geq 2$ ) parametrized by arc length. The curvature of $\sigma$ is the function $k: I \rightarrow$ $R^{+}$of

class $C^{k-2}$ given by : $k(s)=\left\|t^{\prime}(s)\right\|=\left\|\alpha^{\prime \prime}(s)\right\|$.

Clearly $k(s)$ is the curvature of $\sigma$ at the point $\sigma(s)$. We shall say that $\sigma$ is bi regular if $k$ is everywhere nonzero. In this case the radius of curvature of $\sigma$ at the point $\sigma(s)$ is $r(s)=1 / k(s)$.

\section{Remark (3.5):}

If $\sigma: I \rightarrow R^{n}$ is an arbitrary regular parametrized curve, the curvature $k(t)$ of $\sigma$ at the point $\sigma(t)$ is defined by reparametrizing the curve by arc length. If $\sigma_{1}=\sigma^{\circ} s^{-1}$ is a parametrization of $\sigma$ by arc length and $k_{1}$ is the curvature of $\sigma_{1}$ then we define

$k: I \rightarrow \mathrm{R}^{+}$by setting $k(t)=k_{1} s(t)$ so the curvature of $\sigma$ at the point $\sigma(t)$ is equal to the curvature of $\sigma_{1}$ at thepoint $\sigma_{1}(s(t))=$ $\sigma(t) \cdot[12, \mathrm{pp} 15]$

Theorem(3.6):(Beltrami-Enneper)

Every asymptotic curve with curvature $\kappa \neq 0$ and torsion $\tau$ satisfies the equation $\tau^{2}=-K$.

Proof: Let $c(s)$ be an asymptotic curve with $I I\left(c^{\prime}, c^{\prime}\right)=0$. Then the normal curvature of $c$ vanishes . Hence $e_{2}$ is tangential to the surface and consequently the vector $e_{3}=v$ is a unit normal, possibly up to sign. We now calculate $\tau=\left\langle e_{2}^{\prime}, e_{3}\right\rangle=\left\langle e_{2}^{\prime}, v\right\rangle=\operatorname{II}\left(e_{1}, e_{2}\right)$. From this it follows that :

$K=\operatorname{Det} I I / \operatorname{Det} I=I I\left(e_{1}, e_{1}\right) I I\left(e_{2}, e_{2}\right)-\left(I I\left(e_{1}, e_{2}\right)\right)^{2}=0-\tau^{2}$.

[18, pp84]

Definition (3.7): The curvature $\mathrm{e} k$ at each point along an oriented curve $\gamma$ is defined by

$\frac{d T}{a s}=k N ;$ equivalently $\mathrm{k}=\left\langle\frac{d T}{a s}, N\right\rangle .[1, \mathrm{pp} 18]$

The reciprocal $\frac{1}{|k|}$ of the curvature is known as the radius of curvature.

The center of curvature $: \gamma(s)+\frac{1}{|k|} N(s)$

is the center of the circle best approximating the curve at $\gamma(s)$. This best-approximating circleof the curve is known as the osculating circle.[1,pp19]

\section{Proposition (3.8):}

Suppose $\gamma(\mathrm{s})$ is a normal section, parametrized by arc length determined by a unit tangent vector $X_{p} \in T_{p}$ Mand the unit normal vector $N_{p}$. Then the normal curvature of $\gamma(\mathrm{s})$ with respect to $N_{p}$ at $p$ is given by the second fundamental form:

$k\left(X_{p}\right)=\left\langle L\left(X_{p}\right), X_{p}\right\rangle=I I\left(X_{p}, X_{p}\right)$.

Proof: By definition $\gamma(0)=\mathrm{p}$ and $\gamma^{\prime}(0)=X_{p}$. Let $T(s):=\gamma^{\prime}(s)$ be the unit tangent vector field along $\gamma(\mathrm{s})$. Then the curvature of the normal section $\gamma(\mathrm{s})$ is:

$\mathrm{k}\left(\gamma^{\prime}(\mathrm{s})\right)=\left\langle\gamma^{\prime \prime}(s), N_{\gamma(s)}\right\rangle$ 


$$
\begin{gathered}
=\left\langle\frac{d T}{d s}, N_{\gamma(s)}\right\rangle \\
=\left\langle L\left(\gamma^{\prime}(s)\right), T\right\rangle \\
=\langle L(T), T\rangle
\end{gathered}
$$

Evaluating at $s=0$ gives :

$k\left(X_{p}\right)=\left\langle L\left(X_{p}\right), X_{p}\right\rangle=I I\left(X_{p}, X_{p}\right) \cdot[11, \mathrm{pp} 33]$

\section{Definition (3.9):}

Let $k_{1}$ and $k_{2}$ be the principal curvatures of a regular surface $S$ at a point $p$. Define:

i. the Gaussian curvature of $S$ at $p$ as the product $K=k_{1} k_{2}$;

ii. the mean curvature of $S$ at $p$ as the average $H=\frac{k_{1}+k_{2}}{2}$ of the principal curvatures.

[16,pp189]

Theorem(3.10): The mean curvature is also the normal curvature averaged over all directions:

Let $v \theta=\cos (\theta) e_{1}+\sin (\theta) e_{2}$ where $e_{1}, e_{2}$ are an orthonormal basis of $T_{x} M$. Then

$$
\frac{1}{2 \pi} \int_{0}^{2 \pi} k_{n}\left(x, v_{\theta}\right) d \theta=H
$$

Proof: Without loss of generality take $e_{1}=v_{1}$ and $e_{2}=v_{2}$ where $v_{1}, v_{2}$ are the principal directions.(This change of orthogonal basis only amounts to a translation in the angle $\theta \mapsto \theta-\theta_{0}$ which does not change the integral of interest.) we have :

$$
\frac{1}{2 \pi} \int_{0}^{2 \pi} k_{n}\left(x, v_{\theta}\right) d \theta=\frac{k_{1}}{2 \pi} \int_{0}^{2 \pi} \cos ^{2}(\theta) d \theta+\frac{k_{2}}{2 \pi} \int_{0}^{2 \pi} \sin ^{2}(\theta) d \theta=\frac{k_{1}+k_{2}}{2} .
$$

[1,pp77]

\section{RADIUS CURVATURE}

Consider a fixed point $P$ on a given curve and a variable point $Q$ on the same curve.

i. The center of curvature at $P$ is the limit of the intersection of the normal at $P$ and the normal at $Q$, when $Q$ converges to $P$.

ii. The distance between $P$ and the corresponding center of curvature is called the radius of curvature at $P$.

iii. The locus of all the centers of curvature is called the evolute of the given curve. As already mentioned, we take for granted that this definition makes sense which is of course false, even for very good curves! For example if the curve is a straight line the two normal s at $P$ and $Q$ are parallel and you cannot even start the process! Our purpose is therefore once more to guess what a "good" contemporary definition should be. Let us translate Huygens' argument in contemporary terms. [6, pp38]

The "radius of curvature" which is the radius of the osculating circle is defined at each point of the space curve for which $k \neq 0$ as the reciprocal of the curvature i.e. $R_{k}=\frac{1}{k}$. There may be an advantage in using the concept of "curvature" as the principal concept instead of "radius of curvature", that is the curvature is defined at all regular points while the radius of curvature is defined only at the regular points with non-vanishing curvature.[14,pp 44]

Given that a unit of length along the curve path is $\delta s$ and that the tangent line changes its direction over $\delta s$ by an angle $\delta \theta$ where $\theta$ is the angle of the tangent with the $x$ axis then the principal curvature is given by $\mathrm{c}=\frac{\delta \theta}{\delta s}$.

The radius of curvature is simply the inverse of the curvature, or $\rho=1 / \mathrm{c}$. At a point of Inflection of a curve $\mathrm{c}=0$ and $\rho=\infty$. Conversely at a cusp of a curve $\mathrm{c}=\infty$ and $\rho=0 .[4, \mathrm{pp} 11]$

The radius of curvature is simply the inverse of the curvature, or $\rho=1 / c$. At a point of inflection of a curve, $c=0$ and $\rho=\infty$. Conversely, at a cusp of a curve, $c=\infty$ and $\rho=0$. The radius of curvature at lobes of polar curves is of interest in order to define the "tightness" of the lobes. At the peak of the lobe, $r$ ' $=0$ and $\rho=\mathrm{r}^{2} /(\mathrm{r}-\mathrm{r}$ "). This reduces to $\rho=r$ in the case of a circle, for which $r$ " $=0$.For surfaces the geometry underlying the concept of curvature is more complex. Curvature of a surface at a point $\mathrm{p}$ is normally given as the Gaussian curvature $k=k_{1} k_{2}$

where the k's are the principal curvatures with $k_{1}$ being the minimum curvature at $p$ and $k_{2}$ being the maximum curvature at $p$. These curvatures are determined by the two-dimensional curvature of the intersections of the surface with all possible planes containing $p$. If $k_{1}$ and $k_{2}$ are both of the same sign, the point $p$ is an elliptic point and the surface is dome-like at $p$. If $k_{1}$ and $k_{2}$ have opposite signs, the point $p$ is a hyperbolic point and the surface is saddle-like at $p$. If either $k_{1}$ or $k_{2}$ is zero the point $\mathrm{p}$ is a parabolic point. A line separating positive and negative $K$ regions is a parabolic line.[4,pp12]

Definition (4.11): Let $A$ be any subset of $R^{n}$ and $a \in A$.

i. We denote by reach $(A, a)$ the supremum of the real numbers $r$ such that every point of the ball of radius rand center a has an unique projection on $A$ (realizing the distance $d_{A}(m)$ from $m$ to $A$ )reach:

$(A, a)=\sup \left\{r \in R \mid\{p:|p-a|<r\} \subset U_{A}\right\}$.

ii. The reach of $A$ is defined as the infimum of the values of reach $(A, a)$ for $a$ in $A$. We denote by $U_{r}(A)$ the tubular neighborhood of $A$ whose radius is the reach $r$ of $A$. 
[8, pp162]

Lemma (4.12):

If $\beta$ is a unit-speed curve with constant curvature $k>0$ and torsion zero then $\beta$ is part of a circle of radius $1 / \mathrm{k}$.

Proof: Since $\tau=0, \beta$ is a plane curve. What we must now show is thatevery point of $\beta$ is at distance $1 / \mathrm{k}$ from some fixed point which will thus be the center of the circle. Consider the curve $\gamma=\beta+(1 / k) N$. Using the hypothesis on $\beta$ and (as usual) a Frenet formula we find:

$$
\gamma^{\prime}=\beta^{\prime}+\frac{1}{k} N^{\prime}=T+\frac{1}{k}(-k T)=0 .
$$

Hence the curve $\gamma$ is constant that is $\beta(s)+(1 / k) N(s)$ has the same value say $c$ for all $s$. But the distance from $c$ to $\beta(s)$ is: $d(c . \beta(\mathrm{s}))=\|\mathrm{c}-\beta(\mathrm{s})\|=\left\|\frac{1}{k} N(s)\right\|=\frac{1}{k} \cdot[2, \mathrm{pp} 65]$

Proposition (4.13): Suppose $M$ has noumbilic points and $k_{1}$ is constant. Then $M$ is (a subset of) a tube of radius $\mathrm{r}=\frac{1}{\left|k_{1}\right|}$ about a regular curve $\alpha$.

Proof. Choose a principal moving frame $e_{1}, e_{2}$. We have $\omega_{13}=k_{1} \omega_{1}$ and $\omega_{23}=k_{2} \omega_{2}$. Differentiating the first, since $k_{1}$ is constant, we get $\omega_{12} \wedge \omega_{23}=k_{1} \omega_{12} \wedge \omega_{2}$, so $\omega_{12} \wedge\left(k_{2}-k_{1}\right) \omega_{2}=0$. Since $k_{2}-k_{1} \neq 0$,

we infer that $\omega_{12}=\lambda \omega_{2}$ for some scalar function $\lambda$. Now let $\overline{e_{1}}=e_{1}, \overline{e_{2}}, \overline{e_{3}}$ be the Frenet frame of the $e_{1}$ curve. Since both $\omega_{12}=$ 0and $\omega_{13} \neq 0$ when restricted to (pulled back to) ane $e_{1}$ curve we infer that $\cos \theta=0$ and $\theta= \pm \pi / 2$ all along the curve. Then $\bar{\omega}_{23}=$ $\tau \omega_{1}=0$ on the $e_{1}$ curve, so $\tau=0$ and the curve is planar. But then $\omega_{1}=\bar{\omega}_{12}= \pm \bar{\omega}_{13}= \pm k_{1} \bar{\omega}_{1}$, so $k=\left|k_{1}\right|$ is constant and the $e_{1}$ curves are circles.

Now consider,$\alpha=x+\frac{1}{k_{1}} e_{3}$. Then :

$$
d \alpha=d x+\frac{1}{k_{1}} d e_{3}=\omega_{1} e_{1}+\omega_{2} e_{2}+\frac{1}{k_{1}}\left(-k_{1} \omega_{1} e_{1}-k_{2} \omega_{2} e_{2}\right)=\left(1-\frac{k_{2}}{k_{1}}\right) \omega_{2} e_{2} .
$$

so $\alpha$ is constant along the $e_{1}$ curves and $d \boldsymbol{\alpha} \neq \mathbf{0}$ which means that the image of $\alpha$ is a regular curve the center of the tube as desired.[15,pp105]

Proposition (4.14): Consider a plane curve with parametric representation $\mathrm{f}$. In "good cases" the radius of curvature is given by the formula :

$$
\rho=\frac{\left\|f^{\prime}\right\|^{3}}{\left|f_{2}^{\prime} f_{1}^{\prime \prime}-f_{1}^{\prime} f_{2}^{\prime \prime}\right|}
$$

Proof: Let us consider the fixed point $P=f(t 0)$ and the variable point $Q=f(t)$.

The normal vector at $Q$ is thus $n(t)=\left(f_{2}^{\prime}(t)-f_{1}^{\prime}(t)\right)$ and analogously at $P$. The intersection of the two normal $\mathrm{s}$ is thus such that: $f\left(t_{0}\right)+\alpha_{t} n\left(t_{0}\right)=f(t)+\beta_{t} n(t)$ for two scalars $\alpha_{t}$ and $\beta_{t}$ that we have now to determine.

This equality yields the system of equations:

$$
\begin{aligned}
& f_{1}\left(t_{0}\right)+\alpha_{t} f_{2}^{\prime}\left(t_{0}\right)=f_{1}(t)+\beta_{t} f_{2}^{\prime}(t) \\
& f_{2}\left(t_{0}\right)+\alpha_{t} f_{1}^{\prime}\left(t_{0}\right)=f_{2}(t)+\beta_{t} f_{1}^{\prime}(t)
\end{aligned}
$$

so that, by Cramer's rule for solving such a system and the well-known properties of determinants :

When $t$ converges to $t_{0}$, we obtain :

$$
\alpha_{t}=\frac{\operatorname{det}\left(\begin{array}{c}
f_{1}(t)-f_{1}\left(t_{0}\right)-f_{2}^{\prime}(t) \\
f_{2}(t)-f_{2}\left(t_{0}\right) f_{1}^{\prime}(t)
\end{array}\right)}{\operatorname{det}\left(\begin{array}{c}
f_{2}^{\prime}\left(t_{0}\right)-f_{2}^{\prime}(t) \\
-f_{1}^{\prime}\left(t_{0}\right) f_{1}^{\prime}(t)
\end{array}\right)}=\frac{\operatorname{det}\left(\begin{array}{c}
\frac{f_{1}(t)-f_{1}\left(t_{0}\right)}{t-t_{0}}-f_{2}^{\prime}(t) \\
\frac{f_{2}(t)-f_{2}\left(t_{0}\right)}{t-t_{0}} f_{1}^{\prime}(t)
\end{array}\right)}{\operatorname{det}\left(\begin{array}{c}
f_{2}^{\prime}\left(t_{0}\right)-f_{2}^{\prime \prime}\left(t_{0}\right) \\
-f_{1}^{\prime}\left(t_{0}\right) f_{1}^{\prime \prime}\left(t_{0}\right)
\end{array}\right)} .
$$

$$
\alpha=\lim _{t \rightarrow t_{0}} \alpha_{t}=\frac{\operatorname{det}\left(\begin{array}{c}
f_{1}^{\prime}\left(t_{0}\right)-f_{2}^{\prime}\left(t_{0}\right) \\
f_{2}^{\prime}\left(t_{0}\right) f_{1}^{\prime}(t)
\end{array}\right)}{\operatorname{det}\left(\begin{array}{c}
\left.f_{2}^{\prime}\left(t_{0}\right)-f_{2}^{\prime \prime}\left(t_{0}\right)\right) \\
-f_{1}^{\prime}\left(t_{0}\right) f_{1}^{\prime \prime}\left(t_{0}\right)
\end{array}\right)}=\frac{\left\|f^{\prime}\left(t_{0}\right)\right\|^{2}}{\left|f_{2}^{\prime}\left(t_{0}\right) f_{1}^{\prime \prime}\left(t_{0}\right)-f_{1}^{\prime}\left(t_{0}\right) f_{2}^{\prime \prime}\left(t_{0}\right)\right|} .
$$

The center of curvature at $P$ is then the point

$f\left(t_{0}\right)+\alpha n\left(t_{0}\right)$.

The radius of curvature is simply:

$$
\begin{gathered}
\left\|\alpha n\left(t_{0}\right)\right\|=\left|\frac{\left\|f^{\prime}\left(t_{0}\right)\right\|^{2}}{\left|f_{2}^{\prime}\left(t_{0}\right) f_{1}^{\prime \prime}\left(t_{0}\right)-f_{1}^{\prime}\left(t_{0}\right) f_{2}^{\prime \prime}\left(t_{0}\right)\right|} \sqrt{\left(f_{2}^{\prime}\left(t_{0}\right)\right)^{2}+\left(f_{1}^{\prime}\left(t_{0}\right)\right)^{2}}\right| \\
=\frac{\left\|f^{\prime}\left(t_{0}\right)\right\|^{3}}{\left|f_{2}^{\prime}\left(t_{0}\right) f_{1}^{\prime \prime}\left(t_{0}\right)-f_{1}^{\prime}\left(t_{0}\right) f_{2}^{\prime \prime}\left(t_{0}\right)\right|}
\end{gathered}
$$


which is indeed the announced formula.[6,pp 39]

The study of the curvature of a skew curve was initiated by the French mathematician Mongein 1771, thus still before the introduction of parametric equations.

In three dimensional space, the normal to the curve now becomes a normal plane to the curve: the plane perpendicular to the tangent at a given point. Therefore Monge makes the following definitions:

i. The axis of curvature at a point $\mathrm{P}$ of the skew curve is the limit of the intersection of the normal plane at $\mathrm{P}$ and the normal plane at a point $\mathrm{Q}$ of the curve as $\mathrm{Q}$ converges to $\mathrm{P}$.

ii. The radius of curvature at $P$ is the distance between $P$ and the axis of curvature at $P$.

The fact of having an axis of curvature instead of a center of curvature explains in particular why curves with the same curvature can have very different shapes. The orientation of the axis of curvature is generally not constant and its variations in direction affect in an essential way the shape of the curve.

[6,pp46-47]

Proposition (4.15): "Under suitable assumptions" the axis of curvature is perpendicular to the osculating plane and the radius of curvature is the inverse of the curvature.

Proof: Let us work with a parametric representation

$f:] a, b] \rightarrow \mathbf{R}^{3}, t \mapsto f(t)=\left(f_{1}(t), f_{2}(t), f_{3}(t)\right)$

in particular $f^{\prime}(t)$ and $f^{\prime \prime}(t)$ are linearly independent at each point. We study the curvature at $f\left(t_{0}\right)$ and there is no loss of generality in choosing a rectangular system of coordinates with origin $f\left(t_{0}\right)$ and such that $f^{\prime}\left(t_{0}\right)$ is oriented along the third axis. Thus

$f\left(t_{0}\right)=(0,0,0), f^{\prime}\left(t_{0}\right)=\left(0,0, f_{3}^{\prime}\left(t_{0}\right)\right)$.

The normal plane at $f\left(t_{0}\right)$ is thus the plane with equation $z=0$.

The normal plane at $f(t)$ admits the equation :

$f_{1}^{\prime}(t)\left(x-f_{1}(t)\right)+f_{2}^{\prime}(t)\left(y-f_{2}(t)\right)=f_{3}^{\prime}(t)\left(z-f_{3}(t)=0\right.$.

Its intersection with the plane $z=0$ is thus the line with equation

$f_{1}^{\prime}(t)\left(x-f_{1}(t)\right)+f_{2}^{\prime}(t)\left(y-f_{2}(t)\right)=f_{3}^{\prime}(t) f_{3}(t)$.

in the $(x, y)$ plane. Keeping in mind the very special form of the coordinates of $f\left(t_{0}\right)$ and $f^{\prime}\left(t_{0}\right)$ and dividing by $t-t_{0}$ this equation can equivalently be rewritten

as:

$$
\frac{f_{1}^{\prime}(t)-f_{1}^{\prime}\left(t_{0}\right)}{t-t_{0}}\left(x-f_{1}(t)\right)+\frac{f_{2}^{\prime}(t)-f_{2}^{\prime}\left(t_{0}\right)}{t-t_{0}}\left(y-f_{2}(t)\right)=f_{3}^{\prime}(t) \frac{f_{3}^{\prime}(t)-f_{3}^{\prime}\left(t_{0}\right)}{t-t_{0}} .
$$

Passing to the limit when $t$ converges to $t_{0}$ yields $f 1(t 0) x+f 2(t 0) y=\_f 3(t 0) \_2$.Assume now that $f$ is a normal representation. Then $\left\|f^{\prime}\right\|=1$ thus $f_{3}^{\prime}(t)=1$.

Moreover $f^{\prime \prime}$ is perpendicular to $f^{\prime}$ :

$$
f_{1}^{\prime} f_{1}^{\prime \prime}+f_{2}^{\prime} f_{2}^{\prime \prime}+f_{3}^{\prime} f_{3}^{\prime \prime}=0
$$

thus $f_{3}^{\prime \prime}(t)=0$. The system of equations of the axis of curvature can thus be written as :

$$
\left\{\begin{array}{l}
f_{1}^{\prime \prime}\left(t_{0}\right) x+f_{2}^{\prime \prime}\left(t_{0}\right) y+f_{3}^{\prime \prime}\left(t_{0}\right) z=1 \\
\quad z=0
\end{array}\right.
$$

The first plane is orthogonal to $f^{\prime \prime}\left(t_{0}\right)$ and the second one is orthogonal to $f^{\prime}\left(t_{0}\right)$ thus their intersection the axis of curvature is perpendicular to these two vectors which by span the osculating plane.

The radius of curvature is the distance in the $(x, y)$ plane between the origin and the line with equation:

$$
f_{1}^{\prime \prime}\left(t_{0}\right) x+f_{2}^{\prime \prime}\left(t_{0}\right) y=1 \text {. }
$$

This distance is simply:

[6,pp49-50]

$$
\sqrt{\left(f_{1}^{\prime \prime}\left(t_{0}\right)\right)^{2}+\left(f_{2}^{\prime \prime}\left(t_{0}\right)\right)^{2}}=\sqrt{\left(f_{1}^{\prime \prime}\left(t_{0}\right)\right)^{2}\left(f_{2}^{\prime \prime}\left(t_{0}\right)\right)^{2}\left(f_{3}^{\prime \prime}\left(t_{0}\right)\right)^{2}}=\left\|f^{\prime \prime}\left(t_{0}\right)\right\| .
$$

\section{CULULITUING OF RADIUS OF CURVATURE NUMERICLY:}

Example (5.1):

Find the points on the parabola $y^{2}=8 x$ at which the radius of curvature is $\frac{125}{16}$

Solution: $y=2 \sqrt{2} \sqrt{x}$

$$
\begin{gathered}
y_{1}=\frac{\sqrt{2}}{\sqrt{x}}, y_{2}=\frac{-1}{\sqrt{x} x^{3 / 2}} \\
\rho=\frac{\left(1+y_{1}^{2}\right)^{3 / 2}}{\left|y_{2}\right|}=\left(1+\frac{1}{x}\right)^{3 / 2} \cdot \sqrt{2} x^{3 / 2}=\sqrt{2}(x+2)^{3 / 2}
\end{gathered}
$$

Given 
Example (5.2):

$$
\begin{gathered}
\rho=\frac{125}{16} \quad \therefore(x+2)^{3 / 2}=\frac{125}{16 \sqrt{2}}=\left(\frac{5}{2 \sqrt{2}}\right)^{3} \\
\therefore(x+2)^{3 / 2}=\frac{5}{2 \sqrt{2}} \\
\Rightarrow x+2=\frac{25}{8} \quad x=\frac{9}{8} \\
\Rightarrow y^{2}=8\left(\frac{9}{8}\right) \text { i.e. } y=3,-3
\end{gathered}
$$

Hence the points at which the radius of curvature is $\frac{125}{16}$ areare $(9, \mp 3)$.

Find the radius of curvature using Matlab.

\section{Solution:}

$\%$ Find the points on the parabola

clear

clc

closeall

symsyxfy $1 \mathrm{y} 2 \mathrm{p}$

$\mathrm{y} 2=8 * \mathrm{x}$

$\mathrm{f}=\mathrm{y} 2$;

$\mathrm{y}=\operatorname{sqrt}(\mathrm{f})$

$\mathrm{y} 1=\operatorname{sqrt}\left(1+(\mathrm{y} 1)^{\wedge} 2\right) / \operatorname{sqrt}(2)$

$\mathrm{y} 2=-1 / \operatorname{sqrt}\left(\mathrm{x}^{*}(\mathrm{x})^{\wedge} 3 / 2\right)$

$\mathrm{p}=\left(\left(\left(1+(\mathrm{y} 1)^{\wedge} 2\right)\right)^{\wedge} 3 / 2\right) / \mathrm{abs}(\mathrm{y} 2)$

$\mathrm{p}=125 / 16$

$y=-3: 3: 5$

$\mathrm{x}=(\mathrm{y} / 8)$

$\operatorname{plot}(\mathrm{x}, \mathrm{y})$

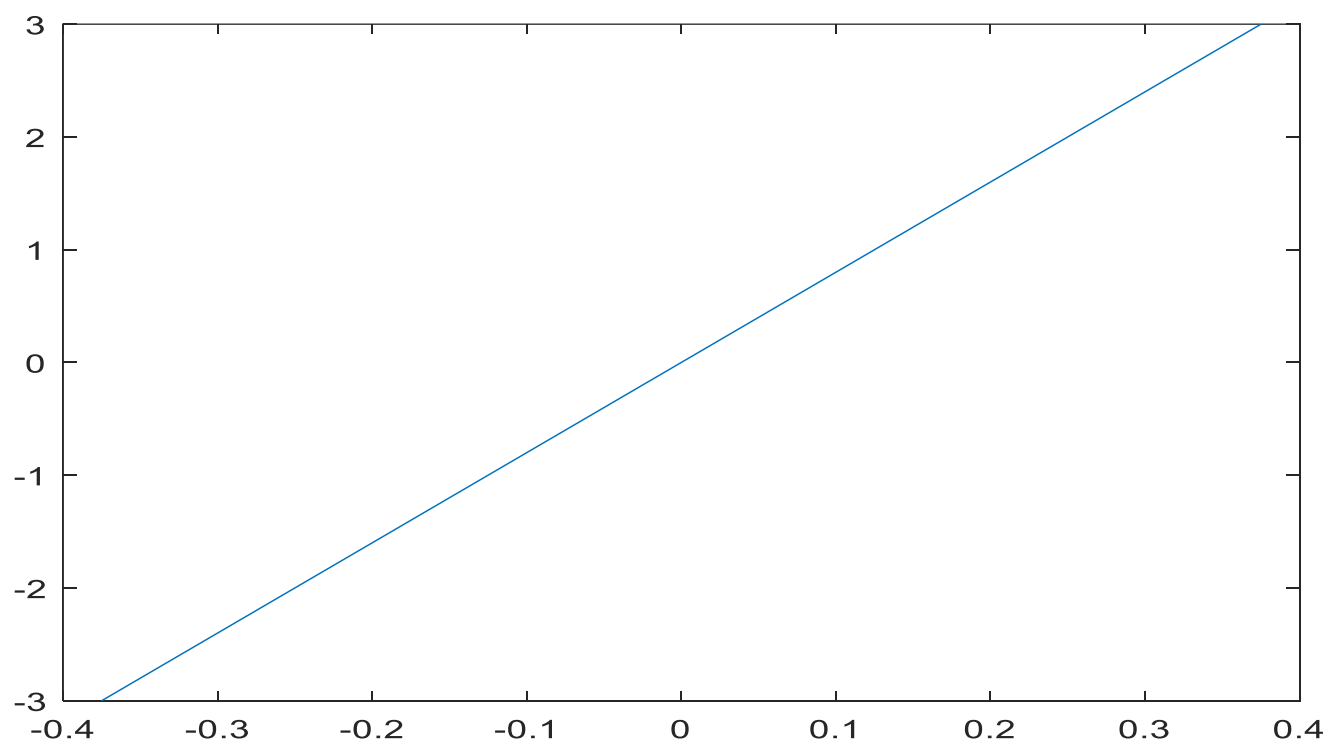

Fig. No. (1): The Matlab Calculating

\section{RESULTS}

After we showed the calculation of Radius Curvature of the Parabola in to diminution using a new mathematical technique we found the following some results :We showed that a new mathematical technique gives us a precise results of high speed compared with that of numerical also we stated the ability capability of graphs or diagram drawing to any radius of curvature via a new mathematical technique we explained the possibility of the calculation of Radius of curvature by a new mathematical technique with a very high rate and accuracy finally we can considered a new mathematical technique as a theory which is considered one of the most important mathematical technique to calculate the Radius of curvature and the other mathematical conceptions . 


\section{CONCLUSION}

Finally we can say that the method which we used in this paper help us in finding the most accurate results and drawing them in a more rapid, attractive and clear way. Therefore we hope that researchers will use this method (A New Mathematical Technique NMT) in their scientific papers.

\section{REFERENCES}

1. Albert Chern, Discrete Differential Geometry, November.19, 2020.pp(18-19,77).

2. Barret O’Neill -Elementary Differential Geometry, MA018303, Burlington, USA-2006.pp(53-54,212)

3. Brian D.Hahn, DarielT.valentine-ESSENTIAL MATLAB for English and scientists. Academic Pres ,2019.pp(3).

4. David H. VonSeggern.CRCStandard Curvesand Surfaceswith Mathematica. CRC press, 2017.pp(12).

5. David Salomon, Curves and Surfacesfor Computer Graphics.Springer Science\&Business Media, Inc2007.pp(30-31).

6. Francis Borceux. A Differential Approachto Geometry. Springer International Publishing Switzerland 2014.pp[38-39,40, 92].

7. Ivo TerekCouto and Alexandre Lymberopoulos. Introduction to Lorentz GeometryCurves and Surfaces. CRC Press is an imprint of Taylor \& Francis Group, LLC.2021.pp(201).

8. Jean-Daniel Boissonnat ,MoniqueTeillaud Effective Computational Geometry for Curvesand Surfaces, Springer Verlag Berlin Heidelberg. 2006.pp(162-163).

9. John Oprea, Differential Geometry and its Applications, The Mathematical Association of America, ISBN 978-0-88385748-9, Printed in the United States of America, Current Printing (last digit): 1098765432 1, 2007 .pp(28).

10. Kristopher Tapp .Differential Geometry of Curves and Surfaces. Berlin ,Sprininger , 2016.pp(62).

11. Loring W. Tu - Differential GeometryConnections, Curvature, and Characteristic Classes . Springer International Publishing AG 2017.pp(33).

12. Marco Abate, Francesca Tovena .Curves and Surfaces. Springer Science, Business Media. 2012. pp(4,15).

13. Sebastian Montiel, Antonio Ros, Curves and Surfaces. American Mathematical Society, 2009.pp(3).

14. Taha Sochi, Principles of Differential Geometry, London, September 9, 2016.pp(25,44).

15. Theodore Shifrin, Differential Geometry A First Course in Curves and Surfaces, Summer, 2016.pp $(15,105)$.

16. Thomas Banchoff ,Stephen Lovett. Differential Geometry of Curves and Surfaces. Taylor \& Francis Group, LLC.2010.pp(25,189).

17. Weiyi. Zhang, Geometry of Curves and Surfaces, Mathematic Institute, University of Warwick, 2019.pp(10-11).

18. Wolfgang Kühnel. Differential Geometry Curves - Surfaces - Manifolds ,Student Math. American Mathematical Society. 2015.pp(84). 